\title{
TITLE:
}

\section{Harmonic Langmuir waves. I. Nonlinear dispersion relation}

$\operatorname{AUTHOR}(S)$ :

Yoon, PH; Gaelzer, R; Umeda, T; Omura, Y; Matsumoto, $\mathrm{H}$

\section{CITATION:}

Yoon, PH ... [et al]. Harmonic Langmuir waves. I. Nonlinear dispersion relation. PHYSICS OF PLASMAS 2003, 10(2): 364-372

\section{ISSUE DATE:}

2003-02

URL:

http://hdl.handle.net/2433/39790

\section{RIGHT:}

Copyright 2003 American Institute of Physics. This article may be downloaded for personal use only. Any other use requires prior permission of the author and the American Institute of Physics. 


\title{
Harmonic Langmuir waves. I. Nonlinear dispersion relation
}

\author{
P. H. Yoon \\ Radio Atmospheric Science Center, Kyoto University, Kyoto, Japan \\ and Institute for Physical Science and Technology, University of Maryland, College Park, Maryland \\ 20742-2431 \\ R. Gaelzer ${ }^{\text {a) }}$ \\ Institute for Physical Science and Technology, University of Maryland, College Park, Maryland 20742-2431 \\ T. Umeda, Y. Omura, and H. Matsumoto \\ Radio Atmospheric Science Center, Kyoto University, Kyoto, Japan
}

(Received 29 August 2002; accepted 19 November 2002)

\begin{abstract}
Generation of electrostatic multiple harmonic Langmuir modes during beam-plasma interaction process has been observed in laboratory and spaceborne active experiments, as well as in computer simulation experiments. Despite earlier efforts, such a phenomenon has not been completely characterized both theoretically and in terms of numerical simulations. This paper is a first in a series of three papers in which analytic expressions for harmonic Langmuir mode dispersion relations are derived and compared against the numerical simulation result. (C) 2003 American Institute of Physics. [DOI: 10.1063/1.1537238]
\end{abstract}

\section{INTRODUCTION}

The beam-plasma interaction is one of the most important problems in plasma physics for both scientific and commercial applications. The weak beam-plasma (or bump-ontail) instability has also played a crucial role as a testbed for various nonlinear plasma turbulence theories, which include quasilinear, ${ }^{1-4}$ weak turbulence, ${ }^{5-14}$ and strong turbulence ${ }^{15-21}$ theories.

One of the intriguing phenomena in the beam-plasma interaction process is the excitation of electrostatic multiple harmonic Langmuir waves, which were first observed in laboratory experiments in the $1960 \mathrm{~s},{ }^{22-30}$ as well as in spacecraft observation $^{31}$ and spaceborne rocket active experiment. ${ }^{32}$ Such a phenomenon was also observed in various numerical computer simulations of the beam-plasma interactions. ${ }^{33-41}$ According to these publications, the harmonic waves are excited at multiples of the plasma frequency,

$$
\omega \sim n \omega_{p e}, \quad n=2,3,4, \ldots
$$

(here, $\omega_{p e}^{2}=4 \pi \hat{n} e^{2} / m_{e}$ is the square of the electron plasma frequency, $\hat{n}, e$, and $m_{e}$ being the ambient density, unit electric charge, and electron rest mass, respectively), and they all propagate with phase speeds roughly equal to the beam propagation speed,

$$
\omega / k \sim V_{0},
$$

where $V_{0}$ is the average electron beam speed. This implies that the wavelengths of the harmonic components, $\lambda_{n}$, become shorter and shorter for higher harmonics, $\lambda_{n}$ $\sim 2 \pi V_{0} /\left(n \omega_{p e}\right)$, or equivalently, the wave number of the

\footnotetext{
${ }^{a)} \mathrm{CNPq}$ fellow. Permanent address: Instituto de Física e Matemática, Universidade Federal de Pelotas (UFPel), Caixa Postal 354, 96010900 Pelotas, RS, Brazil.
}

harmonic with frequency in the vicinity of $n \omega_{p e}$ is given by

$$
k \sim n \omega_{p e} / V_{0} .
$$

Available theories in the literature for the harmonic generation can be categorized into two classes. The first class of theories, which were developed in the early 1970s, view the harmonics as forced electrostatic perturbations generated by the interaction of trapped electrons and large-amplitude coherent Langmuir waves. ${ }^{33,36,42-44}$ Such theories are applicable for a sufficiently strong and cold beam, and for relatively early nonlinear phase where the beam-plasma interaction is dominated by the formation of coherent phasespace vortices.

Another view, recently developed in Ref. 45, is to consider the harmonics as eigenmodes of nonlinear plasma system. This approach was prompted by recent simulations s $^{40,41}$ which show that harmonic modes persist even in the late nonlinear phase when the coherent phase space structure is no longer apparent, and when the plasma has entered a stage which can be genuinely characterized by random phases.

The simulations by Schriver et al. ${ }^{40}$ and Kasaba et al., ${ }^{41}$ which were designed to resolve only up to the first harmonic (i.e., $n=1$ and 2), clearly show on the basis of simulated $\omega-k$ diagram, that the first harmonic mode occupies a broad spectral range which can be best described by a phenomenological dispersion relation,

$$
\omega_{\mathbf{k}}^{L 2} \approx \omega_{p e}\left(2+3 k^{2} \lambda_{\mathrm{De}}^{2} / 2\right),
$$

with a spectrum of $k$ values largely centered around $k$ $\approx 2 \omega_{p e} / V_{0}$. Here, $\lambda_{\mathrm{De}}^{2}=k_{B} T_{e} /\left(4 \pi \hat{n} e^{2}\right)$ is the square of the Debye length.

Clearly, the broadband spectrum and the presistence of the harmonic mode in a fully turbulent stage cannot be discussed solely on the basis of coherent nonlinear dynamics, which is why the alternative theory was developed in Ref. 45. However, as with the simulations in Refs. 40 and 41, the 
original theory in Ref. 45 is also restricted to the first harmonic Langmuir mode only. ${ }^{45,46}$

At present, a fully general theory which combines both aspects of turbulent (i.e., eigenmode theory) and coherent nonlinear dynamics (forced perturbation theory) is not available. The strengths and weaknesses of the two views are as follows: The coherent nonlinear theory has a clearly identifiable mechanism, i.e., particle trapping in the phase-space vortex, which accounts for the generation of harmonic perturbations. On the other hand, it cannot be applied when the system becomes turbulent, that is, when the spectrum broadens, and/or the phase-space vortex is no longer characterized by a coherent wave. In contrast, the incoherent (or, turbulent) eigenmode theory is applicable for a broadband spectrum, and it is capable of explaining the persistence of harmonics over a long period. On the other hand, the theory does not have a self-contained mechanism to generate the initial perturbations for the harmonic modes. Thus in Ref. 46 an arbitrary level of initial harmonic mode had to be imposed. In this respect, it can be envisaged that both the coherent and incoherent nonlinear theories have a role to play in a hypothetical complete theory.

At any rate, the present paper, which is a first in a series of three papers, is motivated by the following two aspects: The analysis of Refs. 45 and 46 pertains only to the first harmonic $(n=2)$. Obviously, it needs to be generalized to all higher harmonics. More importantly, though, the physical derivation of the nonlinear dispersion relation in Refs. 45 and 46 was based upon certain conjectural procedures, which were intended to reproduce the phenomenological dispersion curve, Eq. (1), rather than based upon a systematic deductive method. Since the conceptual foundation of the turbulent eigenmode theory rests upon the very existence of the eigenmode solution itself, it is critical to re-examine the derivation of the harmonic eigenmode solution before we move on further.

The latter objective is the major focus in the present paper (paper I), where a more reliable approximation scheme is devised for the proper derivation of the harmonic Langmuir mode dispersion relation, which is valid for arbitrary harmonics. For the sake of reference, in the second paper, henceforth designated as paper II [R. Gaelzer et al., Phys. Plasmas. 10, 373 (2003)], we will formulate and solve the generalized weak turbulence kinetic equations for the electrons and the waves, utilizing the nonlinear dispersion relation to be derived in the present paper (i.e., paper I). Then, the third paper (paper III) [T. Umeda et al., Phys. Plasmas 10, $382(2003)$ ] is devoted to the Vlasov simulation and comparison with the theory. Although the full description of the simulation will be the focus of paper III, we will also refer to the simulation results in both papers I and II where appropriate.

\section{THEORETICAL FORMULATION}

\section{A. Formal nonlinear dispersion equation}

The starting point of the present analysis is the formal nonlinear spectral balance equation, given by Eq. (3) of Ref. 45 ,

$$
\begin{aligned}
0= & \left(\frac{i}{2} \frac{\partial \epsilon(\kappa)}{\partial \omega} \frac{\partial}{\partial t}+\epsilon(\kappa)\right) I(\kappa) \\
& -2 \int d \kappa^{\prime}\left[\frac{\left|\chi^{(2)}\left(\kappa^{\prime} \mid \kappa-\kappa^{\prime}\right)\right|^{2}}{\epsilon^{*}(\kappa)} I\left(\kappa^{\prime}\right) I\left(\kappa-\kappa^{\prime}\right)\right. \\
& -\left\{\chi^{(2)}\left(\kappa^{\prime} \mid \kappa-\kappa^{\prime}\right)\right\}^{2}\left(\frac{I\left(\kappa-\kappa^{\prime}\right)}{\epsilon\left(\kappa^{\prime}\right)}+\frac{I\left(\kappa^{\prime}\right)}{\epsilon\left(\kappa-\kappa^{\prime}\right)}\right) I(\kappa) \\
& \left.+\bar{\chi}^{(3)}\left(\kappa^{\prime}\left|-\kappa^{\prime}\right| \kappa\right) I\left(\kappa^{\prime}\right) I(\kappa)\right],
\end{aligned}
$$

where $\kappa=(\mathbf{k}, \omega), \quad \kappa^{\prime}=\left(\mathbf{k}^{\prime}, \omega^{\prime}\right), \quad \kappa-\kappa^{\prime}=\left(\mathbf{k}-\mathbf{k}^{\prime}, \omega-\omega^{\prime}\right)$, and $\int d \kappa^{\prime}=\int d \mathbf{k}^{\prime} \int d \omega^{\prime}$.

In Eq. (2),

$$
\epsilon(\kappa)=1+\chi(\kappa)
$$

is the linear plasma response function, and $\chi(\kappa), \chi^{(2)}\left(\kappa^{\prime} \mid \kappa\right.$ $\left.-\kappa^{\prime}\right)$, and $\bar{\chi}^{(3)}\left(\kappa^{\prime}\left|-\kappa^{\prime}\right| \kappa\right)$ are the linear, second-order and third-order susceptibilities, respectively,

$$
\begin{aligned}
\chi(\kappa)=\sum_{a} \frac{\omega_{p a}^{2}}{k^{2}} \int & d \mathbf{v} \mathbf{k} \cdot \mathbf{g}_{\kappa} f_{a}, \\
\chi^{(2)}\left(\kappa^{\prime} \mid \kappa-\kappa^{\prime}\right)= & \frac{-i}{2} \sum_{a} \frac{e_{a}}{m_{a}} \frac{\omega_{p a}^{2}}{k k^{\prime}\left|\mathbf{k}-\mathbf{k}^{\prime}\right|} \\
& \times \int d \mathbf{v}\left\{\left(\mathbf{k}^{\prime} \cdot \mathbf{g}_{\kappa}\right)\left[\left(\mathbf{k}-\mathbf{k}^{\prime}\right) \cdot \mathbf{g}_{\kappa}-\kappa^{\prime} f_{a}\right]\right. \\
& \left.+\left(\kappa^{\prime} \leftrightarrow \kappa^{-} \kappa^{\prime}\right)\right\}, \\
\chi^{(3)}\left(\kappa^{\prime}\left|-\kappa^{\prime}\right| \kappa\right)= & \frac{1}{2} \sum_{a} \frac{e_{a}^{2}}{m_{a}^{2}} \frac{\omega_{p a}^{2}}{k^{2} k^{\prime 2}} \\
& \times \int d \mathbf{v}\left(\mathbf{k}^{\prime} \cdot \mathbf{g}_{\kappa}\right)\left[\left(\mathbf{k}^{\prime} \cdot \mathbf{g}_{\kappa-\kappa^{\prime}}\right)\left(\mathbf{k} \cdot \mathbf{g}_{\kappa} f_{a}\right)\right. \\
& \left.+\left(\kappa \leftrightarrow-\kappa^{\prime}\right)\right],
\end{aligned}
$$

where $f_{a}(\mathbf{v})$ is the velocity distribution function for species $a$ [normalized to unity, $\int d \mathbf{v} f_{a}(\mathbf{v})=1$ ],

$$
\mathbf{g}_{\kappa}=\frac{1}{\omega-\mathbf{k} \cdot \mathbf{v}+i 0} \frac{\partial}{\partial \mathbf{v}},
$$

and the summation $\Sigma_{a}$ is over the particle species, with $\omega_{p a}^{2}=4 \pi \hat{n} e_{a}^{2} / m_{a}$ representing the square of the plasma frequency for species $a=e, i$ ( $e$ and $i$ stand for the electrons and ions). The quantity $I(\kappa)$ is the phase-averaged square of the wave electric field,

$$
I(\kappa)=\left\langle\delta E_{\mathbf{k}, \omega} \delta E_{-\mathbf{k},-\omega}\right\rangle=\left\langle\delta E^{2}\right\rangle_{\mathbf{k}, \omega} .
$$

The derivation of Eq. (2) and approximate forms of the various response functions can be found in Ref. 45, and thus, will not be repeated here. We simply mention that the desired dispersion equation is obtained from the real part of Eq. (2), while the imaginary part leads to the wave kinetic equation. Our focus in this study is mainly on the real part of Eq. (2), although we will also discuss the linear damping/growth rate of the harmonic Langmuir modes by considering only the imaginary part of the linear response of Eq. (2) and ignoring 
nonlinear mode coupling terms. The full consideration of the wave kinetic equation is the focus of paper II.

The total spectral wave intensity, $I(\kappa)$, is given by the sum of individual wave intensity for each normal mode, designated by $\alpha$,

$$
I(\kappa)=\sum_{\alpha} \sum_{\sigma= \pm 1} I_{\alpha}^{\sigma}(\mathbf{k}) \delta\left(\omega-\sigma \omega_{\mathbf{k}}^{\alpha}\right) .
$$

Specifically, we shall adopt $\alpha=L n$ to designate the $n$ th-harmonic Langmuir wave. For $n=1$ (the fundamental Langmuir mode), the customary Bohm-Gross dispersion relation,

$$
\omega_{\mathbf{k}}^{L 1}=\omega_{p e}\left(1+3 k^{2} \lambda_{\mathrm{De}}^{2} / 2\right),
$$

is well known. In the present discussion which pertains to high-frequency response of the plasma, we may ignore the low-frequency ion-sound mode altogether. Here we reiterate that the conjecture on the form of the dispersion relation for L2 mode as represented by Eq. (1 $)^{45,46}$ was influenced by the L1 mode dispersion relation (4), rather than systematically derived. In the subsequent discussion we will remedy this shortcoming, and actually derive the proper form of $L 2$ mode dispersion relation, as well as those of all the higher harmonics.

Let us insert Eq. (3) into the real part of Eq. (2). In doing so, we note that the third-order susceptibility contributes very little to the dispersion relation, since it does not contain the inverse of the linear dielectric response function, as the second-order terms do. Therefore, we will ignore the thirdorder nonlinearity in the discussion of the dispersion relations. The result is

$$
\begin{aligned}
0= & \sum_{n=1,2,3, \ldots} \sum_{\sigma= \pm 1} \operatorname{Re} \epsilon\left(\mathbf{k}, \sigma \omega_{\mathbf{k}}^{L n}\right) I_{L n}^{\sigma}(\mathbf{k}) \delta\left(\omega-\sigma \omega_{\mathbf{k}}^{L n}\right) \\
& +2 \operatorname{Re} \int d \mathbf{k}^{\prime}\left[\sum_{n, n^{\prime}} \sum_{\sigma, \sigma^{\prime}= \pm 1} \frac{2\left\{\chi^{(2)}\left(\mathbf{k}^{\prime}, \sigma^{\prime} \omega_{\mathbf{k}^{\prime}}^{L n^{\prime}} \mid \mathbf{k}-\mathbf{k}^{\prime}, \sigma \omega_{\mathbf{k}}^{L n}-\sigma^{\prime} \omega_{\mathbf{k}^{\prime}}^{L n^{\prime}}\right)\right\}^{2}}{\epsilon\left(\mathbf{k}-\mathbf{k}^{\prime}, \sigma \omega_{\mathbf{k}}^{L n}-\sigma^{\prime} \omega_{\mathbf{k}^{\prime}}^{L n^{\prime}}\right)} I_{L n^{\prime}}^{\sigma^{\prime}}\left(\mathbf{k}^{\prime}\right) I_{L n}^{\sigma}(\mathbf{k}) \delta\left(\omega-\sigma \omega_{\mathbf{k}}^{L n}\right)\right. \\
& \left.-\sum_{n^{\prime}, n^{\prime \prime}} \sum_{\sigma^{\prime}, \sigma^{\prime \prime}= \pm 1} \frac{\left|\chi^{(2)}\left(\mathbf{k}^{\prime}, \sigma^{\prime} \omega_{\mathbf{k}^{\prime}}^{L n^{\prime}} \mid \mathbf{k}-\mathbf{k}^{\prime}, \sigma^{\prime \prime} \omega_{\mathbf{k}-\mathbf{k}^{\prime}}^{L n^{\prime \prime}}\right)\right|^{2}}{\epsilon^{*}\left(\mathbf{k}, \sigma^{\prime} \omega_{\mathbf{k}^{\prime}}^{L n^{\prime}}+\sigma^{\prime \prime} \omega_{\mathbf{k}-\mathbf{k}^{\prime}}^{L n^{\prime \prime}}\right)} I_{L n^{\prime}}^{\sigma^{\prime}}\left(\mathbf{k}^{\prime}\right) I_{L n^{\prime \prime}}^{\sigma^{\prime \prime}}\left(\mathbf{k}-\mathbf{k}^{\prime}\right) \delta\left(\omega-\sigma^{\prime} \omega_{\mathbf{k}^{\prime}}^{L n^{\prime}}-\sigma^{\prime \prime} \omega_{\mathbf{k}-\mathbf{k}^{\prime}}^{L n^{\prime \prime}}\right)\right] .
\end{aligned}
$$

We may consider the above expression as a perturbation series in the power of $I_{\mathbf{k}}^{L n}$, with $n$ as the ordering parameter. Keep in mind that the nonlinear response can balance the linear response only when the inverse of linear response, which appears in the nonlinear term, becomes sufficiently small. This can happen, for instance, when the inverse linear response is of the form,

$$
\frac{1}{\epsilon\left(\mathbf{k}-\mathbf{k}^{\prime}, \omega_{\mathbf{k}}^{L n}-\omega_{\mathbf{k}^{\prime}}^{L(n-1)}\right)},
$$

which can be of significance, since the denominator can become very small when the difference $\omega_{\mathbf{k}}^{L n}-\omega_{\mathbf{k}}^{L(n-1)}$ is on the order of $\omega_{p e}$,

$$
\epsilon\left(\mathbf{k}-\mathbf{k}^{\prime}, \omega_{\mathbf{k}}^{L n}-\omega_{\mathbf{k}^{\prime}}^{L(n-1)}\right) \approx \epsilon\left(\mathbf{k}-\mathbf{k}^{\prime}, \omega_{p e}\right) \sim 0 .
$$

On the other hand, other quantities, $\epsilon\left(\mathbf{k}-\mathbf{k}^{\prime}, \omega_{\mathbf{k}}^{L n}-\omega_{\mathbf{k}^{\prime}}^{L n^{\prime}}\right)$, where $n^{\prime} \neq n-1$, that is, when the argument involves the frequency difference between two harmonics that are not immediately adjacent to each other, can be ignored, since objects such as $\epsilon\left(\mathbf{k}-\mathbf{k}^{\prime}, \omega_{\mathbf{k}}^{L n}-\omega_{\mathbf{k}^{\prime}}^{L(n-2)}\right) \approx \epsilon\left(\mathbf{k}-\mathbf{k}^{\prime}, 2 \omega_{p e}\right)$, are finite.

To the lowest order $(n=1)$, we have the linear dispersion relation for $L 1$ mode,

$$
0=\operatorname{Re} \epsilon\left(\mathbf{k}, \sigma \omega_{\mathbf{k}}^{L 1}\right) I_{L 1}^{\sigma}(\mathbf{k}) .
$$

To the next order $(n=2)$, if we keep the leading nonlinear term with the frequency difference equal to $\sim \omega_{p e}$ in the argument of the denominators, then we have

$$
\begin{aligned}
0= & \operatorname{Re} \epsilon\left(\mathbf{k}, \sigma \omega_{\mathbf{k}}^{L 2}\right) I_{L 2}^{\sigma}(\mathbf{k})+4 \operatorname{Re} \int d \mathbf{k}^{\prime} \\
& \times \sum_{\sigma^{\prime}= \pm 1} \frac{\left\{\chi^{(2)}\left(\mathbf{k}^{\prime}, \sigma^{\prime} \omega_{\mathbf{k}^{\prime}}^{L 1} \mid \mathbf{k}-\mathbf{k}^{\prime}, \sigma \omega_{\mathbf{k}}^{L 2}-\sigma^{\prime} \omega_{\mathbf{k}^{\prime}}^{L 1}\right)\right\}^{2}}{\epsilon\left(\mathbf{k}-\mathbf{k}^{\prime}, \sigma \omega_{\mathbf{k}}^{L 2}-\sigma^{\prime} \omega_{\mathbf{k}^{\prime}}^{L 1}\right)} \\
& \times I_{L 1}^{\sigma^{\prime}}\left(\mathbf{k}^{\prime}\right) I_{L 2}^{\sigma}(\mathbf{k}) .
\end{aligned}
$$

Note that the last term in Eq. (5) does not contribute because the delta function condition cannot be satisfied.

To the next order $(n=3)$, we have

$$
\begin{aligned}
0= & \operatorname{Re} \epsilon\left(\mathbf{k}, \sigma \omega_{\mathbf{k}}^{L 3}\right) I_{L 3}^{\sigma}(\mathbf{k})+4 \operatorname{Re} \int d \mathbf{k}^{\prime} \\
& \times \sum_{\sigma^{\prime}= \pm 1} \frac{\left\{\chi^{(2)}\left(\mathbf{k}^{\prime}, \sigma^{\prime} \omega_{\mathbf{k}^{\prime}}^{L 2} \mid \mathbf{k}-\mathbf{k}^{\prime}, \sigma \omega_{\mathbf{k}}^{L 3}-\sigma^{\prime} \omega_{\mathbf{k}^{\prime}}^{L 2}\right)\right\}^{2}}{\epsilon\left(\mathbf{k}-\mathbf{k}^{\prime}, \sigma \omega_{\mathbf{k}}^{L 3}-\sigma^{\prime} \omega_{\mathbf{k}^{\prime}}^{L 2}\right)} \\
& \times I_{L 2}^{\sigma^{\prime}}\left(\mathbf{k}^{\prime}\right) I_{L 3}^{\sigma}(\mathbf{k}) .
\end{aligned}
$$

In general, we have 


$$
0=\operatorname{Re}\left(\epsilon\left(\mathbf{k}, \sigma \omega_{\mathbf{k}}^{L n}\right)+4 \sum_{\sigma^{\prime}= \pm 1} \int d \mathbf{k}^{\prime} \frac{\left\{\chi^{(2)}\left(\mathbf{k}^{\prime}, \sigma^{\prime} \omega_{\mathbf{k}^{\prime}}^{L(n-1)} \mid \mathbf{k}-\mathbf{k}^{\prime}, \sigma \omega_{\mathbf{k}}^{L n}-\sigma^{\prime} \omega_{\mathbf{k}^{\prime}}^{L(n-1)}\right)\right\}^{2}}{\epsilon\left(\mathbf{k}-\mathbf{k}^{\prime}, \sigma \omega_{\mathbf{k}}^{L n}-\sigma^{\prime} \omega_{\mathbf{k}^{\prime}}^{L(n-1)}\right)} I_{L(n-1)}^{\sigma^{\prime}}\left(\mathbf{k}^{\prime}\right)\right) I_{L n}^{\sigma}(\mathbf{k}) \quad(n>2) .
$$

The second-order susceptibility can be approximated by ${ }^{45}$

$$
\begin{aligned}
& \left\{\chi^{(2)}\left(\mathbf{k}^{\prime}, \sigma^{\prime} \omega_{\mathbf{k}^{\prime}}^{L(n-1)} \mid \mathbf{k}-\mathbf{k}^{\prime}, \sigma \omega_{\mathbf{k}}^{L n}-\sigma^{\prime} \omega_{\mathbf{k}^{\prime}}^{L(n-1)}\right)\right\}^{2} \approx-\frac{1}{4 \omega_{p e}^{4}} \frac{e^{2}}{m_{e}^{2}} A_{\mathbf{k}, \mathbf{k}^{\prime}}^{(n)}, \\
& A_{\mathbf{k}, \mathbf{k}^{\prime}}^{(n)}=\left\{\left[(n-1) k^{2} \mathbf{k}^{\prime}+n k^{\prime 2} \mathbf{k}\right] \cdot\left(\mathbf{k}-\mathbf{k}^{\prime}\right)+n(n-1)\left|\mathbf{k}-\mathbf{k}^{\prime}\right|^{2}\left(\mathbf{k} \cdot \mathbf{k}^{\prime}\right)\right\}^{2}\left\{n^{4}(n-1)^{4} k^{2} k^{\prime 2}\left|\mathbf{k}-\mathbf{k}^{\prime}\right|^{2}\right\}^{-1} .
\end{aligned}
$$

This leads to the following dispersion equation:

$$
\operatorname{Re} \epsilon(\mathbf{k}, \omega)=\frac{e^{2}}{m_{e}^{2} \omega_{p e}^{4}} \operatorname{Re} \int d \mathbf{k}^{\prime} \frac{A_{\mathbf{k}, \mathbf{k}^{\prime}}^{(n)} I_{L(n-1)}^{+}\left(\mathbf{k}^{\prime}\right)}{\epsilon\left(\mathbf{k}-\mathbf{k}^{\prime}, \omega-\omega_{\mathbf{k}^{\prime}}^{L(n-1)}\right)},
$$

where we have replaced the argument $\omega_{\mathbf{k}}^{L n}$ by a generic $\omega$, and have restricted ourselves to the case of $\sigma=\sigma^{\prime}=+1$, without loss of generality. The solution to the above equation will be designated as

$$
\omega=\omega_{\mathbf{k}}^{L n} .
$$

We expect at the outset that $\omega_{\mathbf{k}}^{L n} \approx n \omega_{p e}$.

Following the standard approach, which is applicable to the spectral range over which a given eigenmode is either marginally damped or weakly growing, we employ the assumption that

$$
|\operatorname{Im} \epsilon(\mathbf{k}, \omega)| \ll|\operatorname{Re} \epsilon(\mathbf{k}, \omega)|,
$$

which is equivalent to the assumption that the linear growth/ damping rate, $\gamma$, of the wave is much less than the real frequency, $\omega$,

$$
|\gamma| \equiv\left|\frac{\operatorname{Im} \epsilon(\mathbf{k}, \omega)}{\partial \operatorname{Re} \epsilon(\mathbf{k}, \omega) / \partial \omega}\right| \ll|\omega| .
$$

To justify this assumption, we will later compute the growth/ damping rate of the harmonic modes and show that indeed, the above inequality is satisfied. We assume the same for $\epsilon\left(\mathbf{k}-\mathbf{k}^{\prime}, \omega-\omega_{\mathbf{k}^{\prime}}^{L(n-1)}\right)$.

Under this assumption we may approximate the linear response function by

$$
\epsilon(\mathbf{k}, \omega) \approx 1-\frac{\omega_{p e}^{2}}{\omega^{2}}\left(1+3 k^{2} \lambda_{\mathrm{De}}^{2} \frac{\omega_{p e}^{2}}{\omega^{2}}\right) .
$$

For $\epsilon\left(\mathbf{k}-\mathbf{k}^{\prime}, \omega-\omega_{\mathbf{k}^{\prime}}^{L(n-1)}\right)$, we simply replace the appropriate arguments. Further simplification is possible if we note that the frequency difference, $\omega-\omega_{\mathbf{k}^{\prime}}^{L(n-1)}$, is expected to be of order $\sim \omega_{p e}$. Then, after some straightforward manipulations, Eq. (10) can be shown to reduce to

$$
1 \approx \frac{n^{2}}{2\left(n^{2}-1\right)} \frac{e^{2}}{m_{e}^{2} \omega_{p e}^{3}} \int d \mathbf{k}^{\prime} \frac{A_{\mathbf{k}, \mathbf{k}^{\prime}}^{(n)} I_{L(n-1)}^{+}\left(\mathbf{k}^{\prime}\right)}{\omega-\omega_{\mathbf{k}^{\prime}}^{L(n-1)}-\omega_{\mathbf{k}-\mathbf{k}^{\prime}}^{L 1}},
$$

where $\omega_{\mathbf{k}-\mathbf{k}^{\prime}}^{L 1}$ is the fundamental Langmuir mode dispersion relation (4). This is the desired formal nonlinear dispersion equation to be analyzed in the next section. Before we do that however, we remind the readers of the ad hoc treatment employed in Refs. 45 and 46, which were concerned with only $n=2$. In these references, the denominator was simplified by setting

$$
\omega_{\mathbf{k}^{\prime}}^{L 1} \sim \omega_{p e} \quad \text { and } \quad \omega_{\mathbf{k}-\mathbf{k}^{\prime}}^{L 1} \sim \omega_{\mathbf{k}}^{L 1},
$$

thus approximating

$$
\omega-\omega_{\mathbf{k}^{\prime}}^{L 1}-\omega_{\mathbf{k}-\mathbf{k}^{\prime}}^{L 1} \approx \omega-2 \omega_{p e}-(3 / 2)\left(\omega_{p e} k^{2} \lambda_{\mathrm{De}}^{2}\right) .
$$

This procedure was designed to reproduce the approximate solution for $L_{2}$, given by Eq. (1), but of course, it is not strictly justified. In the present paper, the proper solution to Eq. (12) is obtained according to a procedure with a firmer mathematical and physical basis.

\section{B. Dispersion relations for harmonic Langmuir modes}

We approach in a deductive manner, starting with $n$ $=2$. First we note that for $T_{e} \rightarrow 0$ (or, equivalently, $\lambda_{\mathrm{De}}$ $\rightarrow 0$ ), Eq. (12) can be solved exactly for arbitrary $n$. In taking this limit, we ignore the implicit temperature dependence of the wave intensity. The limit, $T_{e} \rightarrow 0$, is therefore, a mathematical one, rather than a physical assumption. First, for $n$ $=2$, we have the low-temperature limit of Eq. (12),

$$
1=\frac{2 e^{2}}{3 m_{e}^{2} \omega_{p e}^{3}} \frac{1}{\omega-2 \omega_{p e}} \int d \mathbf{k}^{\prime} A_{\mathbf{k}, \mathbf{k}^{\prime}}^{(2)} I_{L 1}^{+}\left(\mathbf{k}^{\prime}\right),
$$

which immediately leads to

$$
\begin{aligned}
& \omega_{\mathbf{k}}^{L 2} \approx \omega_{p e}\left(2+\varepsilon_{\mathbf{k}}^{(2)}\right), \\
& \varepsilon_{\mathbf{k}}^{(2)}=\frac{2}{3} \frac{e^{2}}{m_{e}^{2} \omega_{p e}^{4}} \int d \mathbf{k}^{\prime} A_{\mathbf{k}, \mathbf{k}^{\prime}}^{(2)} I_{L 1}^{+}\left(\mathbf{k}^{\prime}\right) .
\end{aligned}
$$

Note that $\varepsilon_{\mathbf{k}}^{(2)}$ is a small correction term, which can be ignored when $\omega_{\mathbf{k}}^{L 2}$ is inserted to the next-order nonlinear dispersion equation. Moving on to $n=3$, we have

$$
1 \approx \frac{9}{16} \frac{e^{2}}{m_{e}^{2} \omega_{p e}^{3}} \frac{1}{\omega-3 \omega_{p e}} \int d \mathbf{k}^{\prime} A_{\mathbf{k}, \mathbf{k}^{\prime}}^{(3)} I_{L(n-1)}^{+}\left(\mathbf{k}^{\prime}\right),
$$

from which we obtain

$$
\begin{aligned}
\omega_{\mathbf{k}}^{L 3} & \approx \omega_{p e}\left(3+\varepsilon_{\mathbf{k}}^{(3)}\right), \\
\varepsilon_{\mathbf{k}}^{(3)} & =\frac{9}{16} \frac{e^{2}}{m_{e}^{2} \omega_{p e}^{4}} \int d \mathbf{k}^{\prime} A_{\mathbf{k}, \mathbf{k}^{\prime}}^{(3)} I_{L 2}^{+}\left(\mathbf{k}^{\prime}\right) .
\end{aligned}
$$


In general, for arbitrary $n$ and $T_{e}=0$, Eq. (12) reduces to

$$
1 \approx \frac{n^{2}}{2\left(n^{2}-1\right)} \frac{e^{2}}{m_{e}^{2} \omega_{p e}^{3}} \frac{1}{\omega-n \omega_{p e}} \int d \mathbf{k}^{\prime} A_{\mathbf{k}, \mathbf{k}^{\prime}}^{(n)} I_{L(n-1)}^{+}\left(\mathbf{k}^{\prime}\right)
$$

from which, the desired solution for $T_{e}=0$ for arbitrary $n$ is obtained

$$
\begin{aligned}
\omega_{\mathbf{k}}^{L n} & =\omega_{p e}\left(n+\varepsilon_{\mathbf{k}}^{(n)}\right), \\
\varepsilon_{\mathbf{k}}^{(n)} & =\frac{n^{2}}{2\left(n^{2}-1\right)} \frac{e^{2}}{m_{e}^{2} \omega_{p e}^{4}} \int d^{3} \mathbf{k}^{\prime} A_{\mathbf{k}, \mathbf{k}^{\prime}}^{(n)} I_{L(n-1)}\left(\mathbf{k}^{\prime}\right) .
\end{aligned}
$$

To obtain the thermal corrections to the nonlinear dispersion relation (13), let us assume that the full dispersion relation can be expanded in a Taylor series in $\lambda_{\mathrm{De}}^{2}$,

$$
\omega_{\mathbf{k}}^{L n}=\left.\omega_{\mathbf{k}}^{L n}\right|_{\lambda_{\mathrm{De}}^{2}=0}+\left.\frac{\partial \omega_{\mathbf{k}}^{L n}}{\partial \lambda_{\mathrm{De}}^{2}}\right|_{\lambda_{\mathrm{De}}^{2}=0} \lambda_{\mathrm{De}}^{2}+\cdots,
$$

where

$$
\left.\omega_{\mathbf{k}}^{L n}\right|_{\lambda_{\mathrm{De}}^{2}=0}=\omega_{p e}\left(n+\varepsilon_{\mathbf{k}}^{(n)}\right)
$$

is already given by Eq. (13). Higher-order Taylor series coefficients can be obtained by taking successive derivatives of the nonlinear dispersion equation (12). We are only interested in the second-order correction. Let us first consider $n$ $=2$. Taking the derivative with respect to $\lambda_{\mathrm{De}}^{2}$ while ignoring possible $\lambda_{\text {De }}^{2}$ dependence of $I_{L 1}^{+}\left(\mathbf{k}^{\prime}\right)$, we have

$$
\begin{aligned}
0= & \frac{2}{3} \frac{e^{2}}{m_{e}^{2} \omega_{p e}^{3}} \int d \mathbf{k}^{\prime} \frac{A_{\mathbf{k}, \mathbf{k}^{\prime}}^{(2)} I_{L 1}^{+}\left(\mathbf{k}^{\prime}\right)}{\left(\omega-\omega_{\mathbf{k}^{\prime}}^{L 1}-\omega_{\mathbf{k}-\mathbf{k}^{\prime}}^{L 1}\right)^{2}} \\
& \times\left(\frac{\partial \omega}{\partial \lambda_{\mathrm{De}}^{2}}-\frac{3}{2} \omega_{p e} k^{\prime 2}-\frac{3}{2} \omega_{p e}\left|\mathbf{k}-\mathbf{k}^{\prime}\right|^{2}\right) .
\end{aligned}
$$

Setting $\lambda_{\mathrm{De}}^{2}=0$, we have

$$
\begin{aligned}
0= & \frac{2}{3} \frac{e^{2}}{m_{e}^{2} \omega_{p e}^{3}} \int d \mathbf{k}^{\prime} \frac{A_{\mathbf{k}, \mathbf{k}^{\prime}}^{(2)} I_{L 1}^{+}\left(\mathbf{k}^{\prime}\right)}{\left(\omega-2 \omega_{p e}\right)^{2}}\left(\left.\frac{\partial \omega}{\partial \lambda_{\mathrm{De}}^{2}}\right|_{\lambda_{\mathrm{De}}^{2}=0}\right. \\
& \left.-\frac{3}{2} \omega_{p e} k^{2}-3 \omega_{p e}\left(k^{\prime 2}-\mathbf{k} \cdot \mathbf{k}^{\prime}\right)\right)
\end{aligned}
$$

Since the factor $\left(\omega-2 \omega_{p e}\right)^{2}$ can be taken out of the $\mathbf{k}^{\prime}$ integral, after some straightforward algebraic manipulations we see that

$$
\begin{aligned}
& \left.\frac{\partial \omega_{\mathbf{k}}^{L 2}}{\partial \lambda_{\mathrm{De}}^{2}}\right|_{\lambda_{\mathrm{De}}^{2}=0}=\frac{3}{2} \omega_{p e}\left(k^{2}+\frac{2 \theta_{\mathbf{k}}^{(2)}}{\varepsilon_{\mathbf{k}}^{(2)}}\right), \\
& \theta_{\mathbf{k}}^{(2)}=\frac{2}{3} \frac{e^{2}}{m_{e}^{2} \omega_{p e}^{4}} \int d \mathbf{k}^{\prime}\left(k^{\prime 2}-\mathbf{k} \cdot \mathbf{k}^{\prime}\right) A_{\mathbf{k}, \mathbf{k}^{\prime}}^{(2)} I_{L 1}^{+}\left(\mathbf{k}^{\prime}\right),
\end{aligned}
$$

or, equivalently,

$$
\omega_{\mathbf{k}}^{L 2}=\omega_{p e}\left(2+\varepsilon_{\mathbf{k}}^{(2)}+\frac{3}{2} k^{2} \lambda_{\mathrm{De}}^{2}+\frac{3 \theta_{\mathbf{k}}^{(2)}}{\varepsilon_{\mathbf{k}}^{(2)}} \lambda_{\mathrm{De}}^{2}\right) .
$$

Next, we move on to $n=3$,

$$
\begin{aligned}
0= & \frac{9}{16} \frac{e^{2}}{m_{e}^{2} \omega_{p e}^{3}} \int d \mathbf{k}^{\prime} A_{\mathbf{k}, \mathbf{k}^{\prime}}^{(3)} I_{L 2}^{+}\left(\mathbf{k}^{\prime}\right)\left[\left.\frac{\partial \omega}{\partial \lambda_{\mathrm{De}}^{2}}\right|_{\lambda_{\mathrm{De}}^{2}=0}\right. \\
& \left.-\frac{3}{2} \omega_{p e}\left(k^{\prime 2}+\frac{2 \theta_{\mathbf{k}^{\prime}}^{(2)}}{\varepsilon_{\mathbf{k}^{\prime}}^{(2)}}+\left|\mathbf{k}-\mathbf{k}^{\prime}\right|^{2}\right)\right] .
\end{aligned}
$$

From this, we obtain

$$
\begin{aligned}
\left.\frac{\partial \omega_{\mathbf{k}}^{L 3}}{\partial \lambda_{\mathrm{De}}^{2}}\right|_{\lambda_{\mathrm{De}}^{2}=0}=\frac{3}{2} \omega_{p e}\left(k^{2}+\frac{2 \theta_{\mathbf{k}}^{(3)}}{\varepsilon_{\mathbf{k}}^{(3)}}\right), \\
\theta_{\mathbf{k}}^{(3)}=\frac{9}{16} \frac{e^{2}}{m_{e}^{2} \omega_{p e}^{4}} \int d \mathbf{k}^{\prime} A_{\mathbf{k}, \mathbf{k}^{\prime}}^{(3)} I_{L 2}^{+}\left(\mathbf{k}^{\prime}\right) \\
\times\left(k^{\prime 2}-\mathbf{k} \cdot \mathbf{k}^{\prime}+\frac{\theta_{\mathbf{k}^{\prime}}^{(2)}}{\varepsilon_{\mathbf{k}^{\prime}}^{(2)}}\right) .
\end{aligned}
$$

In general we obtain the desired nonlinear dispersion relation for harmonic Langmuir mode for any $n \geqslant 2$,

$$
\omega_{\mathbf{k}}^{L n}=\omega_{p e}\left(n+\varepsilon_{\mathbf{k}}^{(n)}+\frac{3}{2} k^{2} \lambda_{\mathrm{De}}^{2}+\frac{3 \theta_{\mathbf{k}}^{(n)}}{\varepsilon_{\mathbf{k}}^{(n)}} \lambda_{\mathrm{De}}^{2}\right),
$$

where $\varepsilon_{\mathbf{k}}^{(n)}$ is defined in Eq. (13), and

$$
\begin{aligned}
\theta_{\mathbf{k}}^{(n)}= & \frac{n^{2}}{2\left(n^{2}-1\right)} \frac{e^{2}}{m_{e}^{2} \omega_{p e}^{4}} \int d^{3} \mathbf{k}^{\prime} A_{\mathbf{k}, \mathbf{k}^{\prime}}^{(n)} I_{L(n-1)}\left(\mathbf{k}^{\prime}\right) \\
& \times\left(k^{\prime 2}-\mathbf{k} \cdot \mathbf{k}^{\prime}+\frac{\theta_{\mathbf{k}^{\prime}}^{(n-1)}}{\varepsilon_{\mathbf{k}^{\prime}}^{(n-1)}}\right)
\end{aligned}
$$

with $\theta_{\mathbf{k}}^{(1)}=0$.

The result we have obtained, namely, Eq. (14), is quite general but formal. In a time-dependent calculation of the entire array of wave kinetic equations, the instantaneous dispersion relation (14), and the coefficient (15) can be computed at each moment in time by numerical means, using the actual intensity, $I_{L n}(\mathbf{k})$, computed on the basis of the wave kinetic equation. However, for the purpose of illustration, let us consider a specific model for the harmonic Langmuirwave spectra.

The physical situation in mind is that an energetic but tenuous population of beam electrons interact with thermal background plasma to excite primary Langmuir waves $(L 1)$. The enhanced $L 1$ mode is the source of the nonlinearity which leads to the existence and excitation of $L 2$ mode, which in turn leads to $L 3$ mode, and so on. In a onedimensional situation, let us assume that the electrons are initially distributed in velocity space according to the model specified by

$$
f_{e}(v)=\frac{e^{-v^{2} / v_{e}^{2}}}{\pi^{1 / 2} v_{e}}+\frac{n_{b}}{n_{0}} \frac{e^{-\left(v-V_{0}\right)^{2} / v_{b}^{2}}}{\pi^{1 / 2} v_{b}},
$$

where the beam-to-background density ratio is supposed to be small, $n_{b} / n_{0} \ll 1$. The thermal speeds associated with the background and the beam are given by

$$
v_{e}=\sqrt{2 k_{B} T_{e} / m_{e}}, \quad v_{b}=\sqrt{2 k_{B} T_{b} / m_{e}},
$$


respectively, where $k_{B}$ is the Boltzmann constant. The quantity $V_{0}$ represents the average beam speed, which we have encountered already. The beam electrons will excite primary (L1) Langmuir waves in a range of spectrum with average wave number given approximately by $k_{0} \approx \omega_{p e} / V_{0}$.

Simulations $^{33-41}$ as well as experiments ${ }^{22-29,32}$ provide evidence that the primary $L 1$ mode gives rise to the secondary excitation of the $L 2$ mode with characteristic wave number equal to $\sim 2 k_{0}$, and that in general, $n$ th-harmonic Langmuir mode $(L n)$ possesses a spectrum with average wave number located at roughly

$$
n k_{0} \approx n \omega_{p e} / V_{0} .
$$

On the basis of this consideration, let us model the onedimensional harmonic Langmuir mode spectra by

$$
I_{L n}(k)=I_{n}\left(\pi^{1 / 2} \delta\right)^{-1} e^{-\left(k-n k_{0}\right)^{2} / \delta^{2}},
$$

where $I_{n}=\int d k I_{L n}(k)$, and $\delta$ represents the spread associated with the spectra. With the above model spectra, we may explicitly evaluate the quantity $\varepsilon_{k}^{(n)}$ as follows:

$$
\begin{aligned}
\varepsilon_{k}^{(n)}= & \frac{n^{2}}{2\left(n^{2}-1\right)} \frac{e^{2} I_{n-1}}{m_{e}^{2} \omega_{p e}^{4}} \\
& \times\left(a_{n} k^{2}-(n-1) b_{n} k_{0} k+(n-1)^{2} c_{n} k_{0}^{2}+c_{n} \frac{\delta^{2}}{2}\right),
\end{aligned}
$$

where

$$
a_{n}=\frac{(n+1)^{2}}{n^{4}(n-1)^{2}}, \quad b_{n}=\frac{2(n+1)(n-2)}{n^{3}(n-1)^{3}}, \quad c_{n}=\frac{(n-2)^{2}}{n^{2}(n-1)^{4}} .
$$

Let us assume that the width of the spectrum, $\delta$, for each $n$, although broad enough to justify the use of incoherent turbulence theory, is sufficiently narrow in comparison with the central wave number, $n k_{0}$, i.e., $\delta<n k_{0}$. Then we may compute the coefficient, $\varepsilon_{k}^{(n)}$, by ignoring terms proportional to $\delta$. As a matter of fact, however, this approximation is quite valid even if $\delta$ is not so small when compared with $k_{0}$, since the coefficient $c_{n}$ is much smaller than $a_{n}$ or $b_{n}$ for a given $n$ (first few members of these coefficients are $a_{2}=0.5625, b_{2}$ $=c_{2}=0 ; \quad a_{3}=0.0494, \quad b_{3}=0.0370, \quad c_{3}=0.0069 ; \quad a_{4}$ $=0.0109, \quad b_{4}=0.0116, \quad c_{4}=0.0031 ; \quad a_{5}=0.0036, \quad b_{5}$ $\left.=0.0045, c_{5}=0.0014 ; \ldots\right)$. Under this assumption, one may also show that $\theta_{k}^{(n)}$ is related to $\varepsilon_{k}^{(n)}$ by

$$
\theta_{k}^{(n)}=\varepsilon_{k}^{(n)}(n-1) k_{0}\left(\frac{n k_{0}}{2}-k\right) .
$$

Making use of these results, we obtain the following specific form of dispersion relation for the harmonic Langmuir modes in a beam-plasma system:

$$
\frac{\omega_{k}^{L n}}{\omega_{p e}}=n+\frac{3}{2} k^{2} \lambda_{\mathrm{De}}^{2}-\frac{3(n-1)}{2}\left(\frac{k}{k_{0}}-\frac{n}{2}\right) \frac{k_{0}^{2} v_{e}^{2}}{\omega_{p e}^{2}}+\varepsilon_{k}^{(n)},
$$

where $k_{0} \approx \omega_{p e} / V_{0}$. In the above, the nonlinear frequency shift factor $\varepsilon_{k}^{(n)}$ can be ignored for all practical purposes, since it is a small quantity.

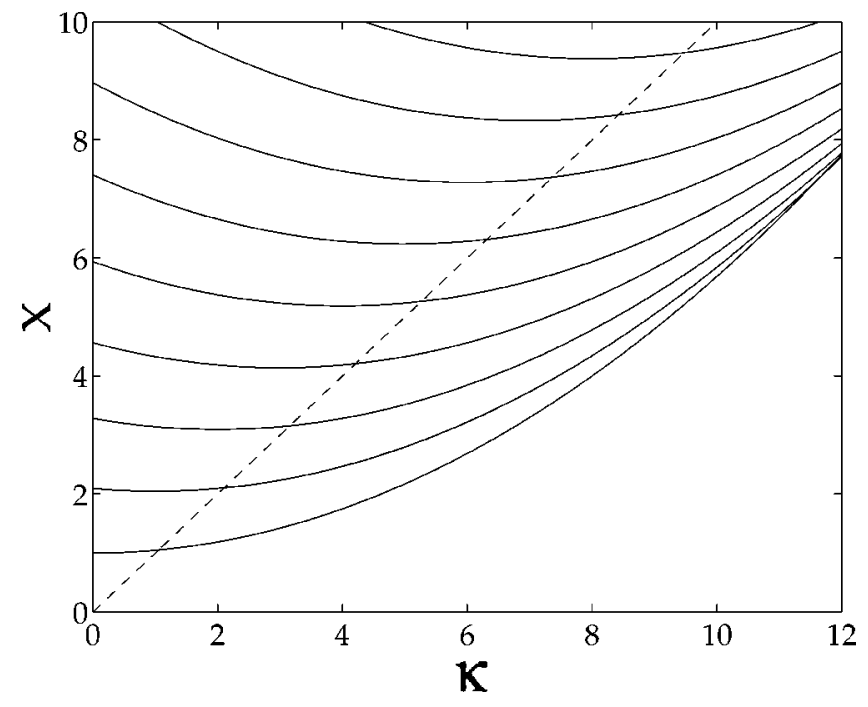

FIG. 1. Normalized frequency $x=\omega / \omega_{p e}$ versus wave number $\kappa$ $=k V_{0} / \omega_{p e}$, for $U=V_{0} / v_{e}=4$. The straight diagonal dashed line is the beam mode, $x=\kappa$ (or equivalently, $\omega=k V_{0}$ ), shown here only as a reference. The growth of each harmonic is expected to occur only around the intersection points between the beam line and the dispersion curves (see the next Fig. 2).

\section{NUMERICAL RESULTS}

Figure 1 shows the plot of analytical dispersion curves for the harmonics when the beam is characterized by $V_{0} / v_{e}=4$. The normalizations for the frequency, wave number, and the ratio of average to background thermal speeds are according to

$$
x=\omega / \omega_{p e}, \quad \kappa=k V_{0} / \omega_{p e}, \quad U=V_{0} / v_{e} .
$$

The straight diagonal dashed line represents the beam mode, $\omega \approx k V_{0}$, or equivalently, $x=\kappa$. The beam mode is not an actual eigenmode solution, but is shown here only as a reference to guide the readers. The growth of each harmonic is expected to occur only around the intersection points between the harmonic mode dispersion curves and the straight beam line. The stacked curves are the eigenmode solutions, written in normalized form, by

$$
x=n+\frac{3 \kappa^{2}}{4 U^{2}}-\frac{3(n-1)}{2 U^{2}}\left(\kappa-\frac{n}{2}\right), \quad n=1,2,3, \ldots .
$$

A caveat in the interpretation of Fig. 1 is that we are only interested in each harmonic dispersion curve which satisfies the condition, $\omega \sim n \omega_{p e}$, a presumption imposed at the outset. Therefore, solutions which do not satisfy this criterion must not be taken literally. A close examination reveals that the dispersion curve for a given $n$ satisfies this criterion only around the vicinity of the intersection point between the dashed beam line and the dispersion curve itself.

Before we close, we note that if we are only interested in the sufficiently early (i.e., quasilinear) phase of the harmonic mode generation process, then we may discuss the amplification of $L n$ mode by considering its "linear" growth rate [which can be obtained by only considering the linear response of Eq. (2)], 


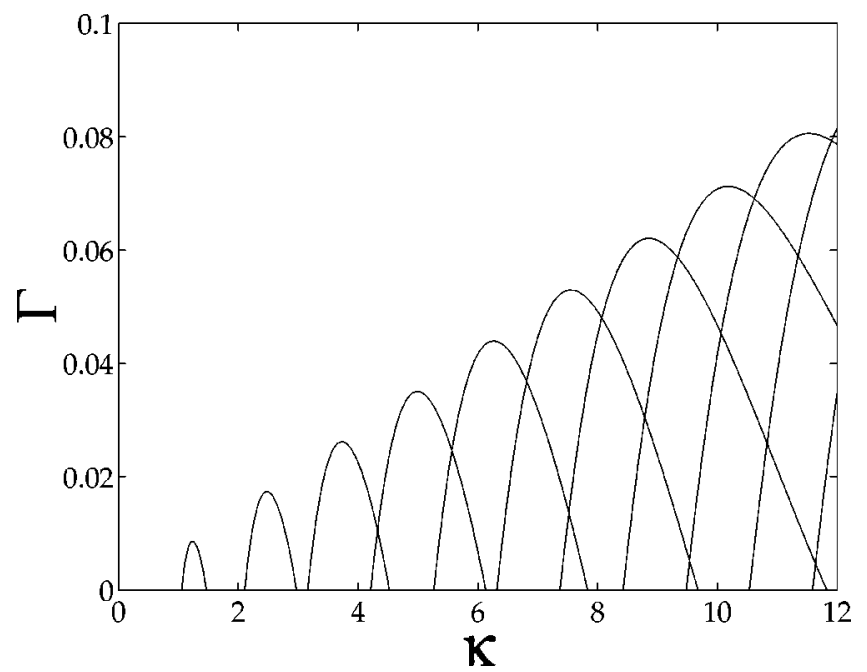

FIG. 2. Normalized growth rate $\Gamma=\gamma / \omega_{p e}$ versus $\kappa$, for $n_{b} / n_{0}=10^{-3}$, $V_{0} / v_{e}=4$, and $T_{e}=T_{b}$.

$$
\begin{aligned}
\gamma= & -\pi^{1 / 2}\left(\frac{\omega_{k}^{L n}}{k v_{e}}\right)^{3}\left[\omega_{k}^{L n} \exp \left(-\frac{\left(\omega_{k}^{L n}\right)^{2}}{k^{2} v_{e}^{2}}\right)\right. \\
& \left.+\frac{n_{b}}{n_{0}}\left(\frac{T_{e}}{T_{b}}\right)^{3 / 2}\left(\omega_{k}^{L n}-k V_{0}\right) \exp \left(-\frac{\left(\omega_{k}^{L n}-k V_{0}\right)^{2}}{k^{2} v_{b}^{2}}\right)\right] .
\end{aligned}
$$
where

Figure 2 plots the normalized growth rate, $\Gamma=\gamma / \omega_{p e}$,

$$
\begin{aligned}
\Gamma= & -\sqrt{\frac{\pi}{2}(x U)^{3}}\left[x \exp \left(-\frac{(x U)^{2}}{2 \kappa^{2}}\right)\right. \\
& \left.+\delta \tau^{3 / 2}(x-\kappa) \exp \left(-\frac{(x-\kappa)^{2} U^{2} \tau}{2 \kappa^{2}}\right)\right],
\end{aligned}
$$

versus $\kappa$, for $\delta=n_{b} / n_{0}=10^{-3}, \quad U=V_{0} / v_{e}=4$, and $\tau$ $=T_{e} / T_{b}=1$. Note that the peak value of $\Gamma$ monotonically increases for increasing $n$, which confirms the findings in Ref. 36 that the initial growth rate for the harmonic mode is higher than that of the fundamental by a factor roughly proportional to the harmonic mode number. (Such a finding is also confirmed by our own simulations as will be discussed in paper III.) A close examination reveals that indeed, the growth of the harmonic mode occurs only in the vicinity of the intersections between the beam mode line and the dispersion curves.

Since the maximum of the normalized growth rate, $\Gamma$, increases for increasing harmonic mode number, $n$, one might wonder whether the original assumption, $\gamma \ll \omega$, is violated for very high $n$. However, since the normalized frequency, $x$, also increases as a function of $n$, the ratio, $\Gamma / x$, always remains small for all $n$. To see this, we replot the result shown in Fig. 2 in a new format, in terms of the ratio, $\Gamma / x=\gamma / \omega$, in Fig. 3. The numerical result reconfirms the inequality, $\gamma \ll \omega$, which validates the present approximate analysis.

It should be noted that when the growth rate formula (21) is plotted in $G=\gamma / \omega_{k}^{L n}$ versus $q=k v_{e} / \omega_{k}^{L n}$, then all the

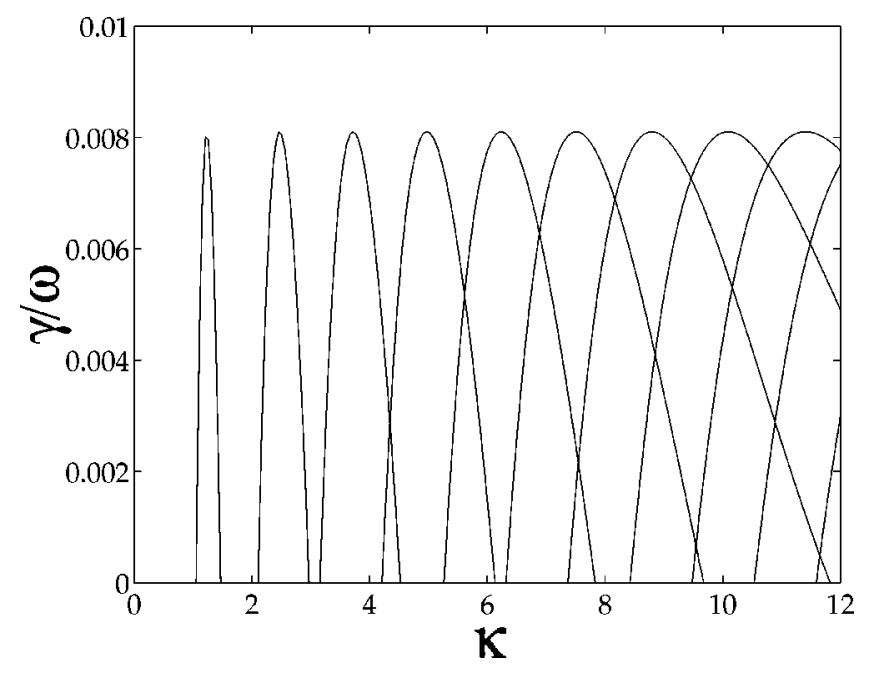

FIG. 3. The ratio of the growth rate to the real frequency, $\gamma / \omega$ versus $\kappa$, for the same set of parameters as in Fig. 2.

curves collapse onto a single curve. This can be seen easily by the fact that Eq. (21) can be expressed in terms of $G$ and $q$ as

$$
\begin{aligned}
G= & -\frac{\sqrt{\pi}}{q^{3}}\left[e^{-1 / q^{2}}+\frac{n_{b}}{n_{0}} \frac{T_{e}^{3 / 2}}{T_{b}^{3 / 2}}\right. \\
& \left.\times\left(1-\frac{q V_{0}}{v_{e}}\right) e^{-\left(v_{e} / v_{b}\right)\left(1 / q-V_{0} / v_{b}\right)^{2}}\right] .
\end{aligned}
$$

To test the idea of the present nonlinear eigenmode theory of harmonic generation, we have also performed a one-dimensional electrostatic Vlasov simulation. The full details of the simulation technique and the in-depth analysis of the results will be the focus of paper III, but here we preview one of the crucial results. The simulation result shown in Fig. 4 corresponds to the intensity of the waves plotted in grayscale format against normalized frequency and wave number,

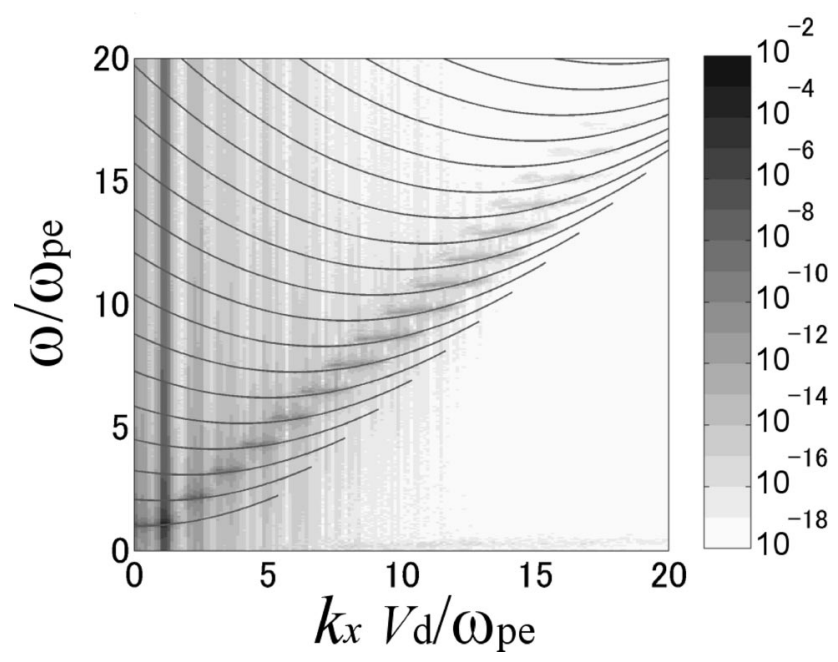

FIG. 4. Simulated dispersion diagram for harmonic Langmuir waves. The intensity of the waves are Fourier analyzed in both space and time, and the result is plotted in grayscale format against normalized frequency and wave number, $\omega / \omega_{p e}$ and $k V_{0} / \omega_{p e}$. We have superposed the theoretical dispersion relation curves (18). 
$\omega / \omega_{p e}$ and $k V_{0} / \omega_{p e}$. The input parameters for the simulation were $n_{b} / n_{0}=10^{-3}, V_{0}=3.5 v_{e}$, and $T_{e} / T_{b}=4$. The Fourier transformation is performed over the simulated data in both space and time, and the result is a simulated $\omega-k$ dispersion diagram shown in Fig. 4. We have superposed the theoretical dispersion relation curves, given by Eq. (18) or (20), on top of the numerically generated wave intensity versus $\omega$ and $k$. The result is the comparison between the theory and simulation.

In Fig. 4, we have made one adjustment to the theoretical curve. Note that the theoretical dispersion curve (18) assumes that the central wave number associated with the fundamental Langmuir mode $(L 1)$ is given by

$$
k_{0}=\omega_{p e} / V_{0} \text {. }
$$

However, this estimation is only a crude one. This stems from the notion that the optimum interaction between the waves and the electrons occur at $v=V_{0}$. In reality, however, the instability takes place when the derivative of the beam distribution is positive, not at the peak of the distribution itself. The maximum positive derivative occurs when $\partial^{2} f_{e} / \partial v^{2}=0$. Considering only the beam distribution into account, one can easily compute

$$
\begin{aligned}
0 & =\left(\partial^{2} / \partial v^{2}\right) \exp \left[-\left(v-V_{0}\right)^{2} / v_{b}^{2}\right] \\
& =-\left(2 / v_{b}^{2}\right)\left[\left(1-2\left(v-V_{0}\right)^{2} / v_{b}^{2}\right] \exp \left[-\left(v-V_{0}\right)^{2} / v_{b}^{2}\right] .\right.
\end{aligned}
$$

This shows that the central wave number associated with $L_{1}$ mode should not be at $\omega_{p e} / V_{0}$, but rather at

$$
k_{0}=\omega_{p e} /\left(V_{0}-v_{b} / \sqrt{2}\right) .
$$

For the input parameters $V_{0}=3.5 v_{e}$ and $T_{e} / T_{b}=4$, we find that $V_{0}^{\text {eff }}=V_{0}-v_{b} / \sqrt{2}=V_{0}-\sqrt{k_{B} T_{b} / m_{e}}=3.2 v_{e}$. Thus, the normalized theoretical curve (20) was computed with $V_{0}^{\text {eff }}$ $=3.2 v_{e}$, instead of $3.5 v_{e}$. The result is an excellent agreement between the simulation result and theory, especially for relatively low harmonics. For higher harmonics, the discrepancy between the theoretical curves and the simulated result becomes more and more apparent, albeit only slightly, as the harmonic mode number increases.

Here, we note that the wave levels fall off in intensity at high harmonics. At first sight, this might seem to contradict the theoretical growth rate prediction which indicates that $\gamma / \omega$ is roughly constant. However, the linear growth rate is only an indication of how rapidly the mode amplifies, but says nothing about the saturation amplitude. The reason for the low saturation amplitudes is their low initial noise amplitude. Even though higher-harmonic modes may grow faster than the lower-harmonic modes in accordance with the linear growth rate prediction, they saturate at low amplitudes since they have to grow from lower initial wave intensity level. In short, Fig. 4 proves that the overall agreement between the theory and simulation is excellent, and that the analytical dispersion relations for the harmonic modes [Eq. (18)] are valid.

\section{CONCLUSIONS AND DISCUSSION}

To conclude this paper, an analytical expression for the harmonic Langmuir mode dispersion relation valid for arbi- trary harmonic $n$ has been derived. The theoretical expression is then compared against the simulated dispersion relation. The result shows an excellent agreement between the theory and simulation, which lends a strong support for treating the electrostatic harmonics as nonlinear eigenmodes of a turbulent plasma. These eigenmodes exist as a result of taking the nonlinear mode coupling associated with finite (but not necessarily large amplitude) waves into account. Such a finding cannot be discussed on the basis of the traditional weak turbulence theory since the traditional theory only considers the primary Langmuir mode $(L 1)$, which is a linear eigenmode.

We have also computed the initial growth rates of the harmonic modes. The focus of the present paper was to characterize the dispersion relation of the harmonic Langmuir modes. Paper II of the present series will make use of the results derived in this paper, to formulate and solve the entire wave kinetic equation as well as the particle kinetic equation in a self-consistent manner. Such an approach is needed if one is to discuss the actual level of the saturated harmonic Langmuir mode spectra. Finally, paper III of the present series presents a detailed account of the Vlasov simulations, one of the results of which has already been showcased in Fig. 4.

\section{ACKNOWLEDGMENTS}

The research carried out at the University of Maryland was supported by Department of Energy (DOE) Grant No. DE-FG02-00ER54584. R.G. acknowledges support from the Brazilian agencies Conselho Nacional de Desenvolvimento Científico e Tecnológico $(\mathrm{CNPq})$ and Fundação de Amparo à Pesquisa do Estado do Rio Grande do Sul (FAPERGS). The work by T.U. was supported by grant-in-aid 03821 for research fellows of the Japan Society for the Promotion of Science (JSPS).

\footnotetext{
${ }^{1}$ A. A. Vedenov, E. P. Velikhov, and R. Z. Sagdeev, Nucl. Fusion 1, 82 (1961).

${ }^{2}$ A. A. Vedenov, E. P. Velikhov, and R. Z. Sagdeev, Nucl. Fusion Suppl. 2, 465 (1962).

${ }^{3}$ W. E. Drummond and D. Pines, Nucl. Fusion Suppl. 3, 1049 (1962)

${ }^{4}$ K. Appert, T. M. Tran, and J. Vaclavik, Phys. Rev. Lett. 37, 502 (1976).

${ }^{5}$ B. B. Kadomtsev and V. I. Petviashvili, Sov. Phys. JETP 16, 1578 (1963).

${ }^{6}$ L. M. Kovrizhnykh, Sov. Phys. JETP 21, 744 (1965).

${ }^{7}$ B. B. Kadomtsev, Plasma Turbulence (Academic, New York, 1965).

${ }^{8}$ V. N. Tsytovich, Sov. Phys. Usp. 9, 805 (1967); Nonlinear Effects in a Plasma (Plenum, New York, 1970); An Introduction to the Theory of Plasma Turbulence (Pergamon, New York, 1977).

${ }^{9}$ A. Rogister and C. Oberman, J. Plasma Phys. 3, 119 (1969).

${ }^{10}$ R. Z. Sagdeev and A. A. Galeev, Nonlinear Plasma Theory (Benjamin, New York, 1969).

${ }^{11}$ C. T. Dum and R. N. Sudan, Phys. Fluids 14, 414 (1971).

${ }^{12}$ R. C. Davidson, Methods in Nonlinear Plasma Theory (Academic, New York, 1972).

${ }^{13}$ A. I. Akhiezer, I. A. Akhiezer, R. V. Polovin, A. G. Sitenko, and K. N. Stepanov, Plasma Electrodynamics (Pergamon, New York, 1975), Vol. 2.

${ }^{14}$ A. G. Sitenko, Fluctuations and Nonlinear Wave Interactions in Plasmas (Pergamon, New York, 1982).

${ }^{15}$ V. E. Zakharov, Sov. Phys. JETP 35, 908 (1972).

${ }^{16}$ A. A. Galeev, R. Z. Sagdeev, Yu. S. Sigov, V. D. Shapiro, and V. I. Shevchenko, Sov. J. Plasma Phys. 1, 5 (1975).

${ }^{17}$ K. Papadopoulos, Phys. Fluids 18, 1769 (1975).

${ }^{18}$ H. L. Rowland and K. Papadopoulos, Phys. Rev. Lett. 39, 1276 (1977).
} 
${ }^{19}$ H. P. Freund, I. Haber, P. Palmadesso, and K. Papdopoulos, Phys. Fluids 23, 518 (1980).

${ }^{20}$ M. V. Goldman, Rev. Mod. Phys. 56, 709 (1984).

${ }^{21}$ P. A. Robinson, Rev. Mod. Phys. 69, 507 (1997).

${ }^{22}$ J. R. Apel, Phys. Rev. Lett. 19, 744 (1967).

${ }^{23}$ J. R. Apel, Phys. Fluids 12, 640 (1969).

${ }^{24}$ J. H. Malmberg and C. B. Wharton, Phys. Fluids 12, 2600 (1969).

${ }^{25}$ K. Mizuno and S. Tanaka, Phys. Rev. Lett. 29, 45 (1972).

${ }^{26}$ K. W. Gentle and J. Lohr, Phys. Fluids 16, 1464 (1973).

${ }^{27}$ H. Mori, J. Phys. Soc. Jpn. 35, 592 (1973).

${ }^{28}$ M. Seidl, W. Carr, D. Boyd, and R. Jones, Phys. Fluids 19, 78 (1976)

${ }^{29}$ R. W. Boswell and P. J. Kellogg, Geophys. Res. Lett. 10, 565 (1983).

${ }^{30}$ X. Llobet, W. Bernstein, and A. Konradi, J. Geophys. Res. 90, 5187 (1985).

${ }^{31}$ I. H. Cairns, J. Geophys. Res. 91, 2975 (1986).

${ }^{32}$ P. J. Kellogg, S. J. Monson, W. Bernstein, and B. A. Whalen, J. Geophys. Res. 91, 12065 (1986)

${ }^{33}$ G. Joyce, G. Knorr, and T. Burns, Phys. Fluids 14, 797 (1971).

${ }^{34}$ B. Goldstein, W. Carr, B. Rosen, and M. Seidl, Phys. Fluids 21, 1569 (1978).
${ }^{35}$ P. L. Pritchett and J. M. Dawson, Phys. Fluids 26, 1114 (1983).

${ }^{36}$ A. J. Klimas, J. Geophys. Res. 88, 9081 (1983); J. Geophys. Res. 95, 14905 (1990).

${ }^{37}$ K. Akimoto, H. L. Rowland, and K. Papadopoulos, Phys. Fluids 31, 2185 (1988).

${ }^{38}$ K.-I. Nishikawa and I. H. Cairns, J. Geophys. Res. 96, 19343 (1991).

${ }^{39}$ L. Yin, M. Ashour-Abdalla, M. El-Alaoui, J. M. Bosqued, and J. L. Bougeret, J. Geophys. Res. 103, 29619 (1998).

${ }^{40}$ D. Schriver, M. Ashour-Abdalla, V. Sotnikov, P. Hellinger, V. Fiala, and A. Mangeney, J. Geophys. Res. 105, 12919 (2000).

${ }^{41}$ Y. Kasaba, H. Matsumoto, and Y. Omura, J. Geophys. Res. 106, 18693 (2001).

${ }^{42}$ W. M. Manheimer, Phys. Fluids 14, 579 (1971).

${ }^{43}$ T. M. O’Neil, J. H. Winfrey, and J. H. Malmberg, Phys. Fluids 14, 1204 (1971).

${ }^{44}$ T. M. O'Neil and J. H. Winfrey, Phys. Fluids 15, 1514 (1972).

${ }^{45}$ P. H. Yoon, Phys. Plasmas 7, 4858 (2000).

${ }^{46}$ R. Gaelzer, L. F. Ziebell, and P. H. Yoon, Phys. Plasmas 9, 96 (2002). 